\title{
Approximate Solution of Schrodinger Equation for Hulthen Potential plus Eckart Potential with Centrifugal Term in terms of Finite Romanovski Polynomials
}

\author{
Cari and Suparmi
}

\begin{abstract}
The energy eigenvalues and eigenfunctions of Schrodinger equation for Hulthen potential plus Eckart potential with the centrifugal term are investigated approximately in terms of finite Romanovski polynomial. The bound state energy eigenvalues are given in a closed form and corresponding eigenfunctions are obtained in terms of Romanovski polynomials.
\end{abstract}

Index Terms-Schrodinger equation, hulthen potential plus eckart potential, centrifugal term, finite romanovski polynomials.

\section{INTRODUCTION}

It is well known that the exact solution of one dimensional Schrodinger equation for some physical potentials are very important since they provide all necessary information for quantum system under consideration. Recently, conciderably efforts have been paid to obtain the exact solution of the central potentials. These potentials include Scarf, Manning Rosen, Rosen Morse, Hulthen, and Wood Saxon potentials. The bound state energy spectra of these potentials have been investigated by various techniques such as factorization methods,[1]-[3] supersymmetric quantum mechanics, [4]-[6] hypergeometric type equation, [7], [8] Nikiforov-Uvarov method,[9]-[11] etc. The exact solution of Schrodinger equation is obtained if the the angular momentum $l=0$. However, for $l \neq 0$, the Schrodinger equation can only be solved approximately for different suitable approximation scheme. One of the suitable approximation scheme is conventionally proposed by Greene and Aldrich. [12]

In this paper we will attempt to solve the Schrodinger equation for Hulthen plus Eckart potential [13], [14] including the centrifugal term in term of finite Romanovski polynomials. The Romanovski polynomials, to be persued by us here, consist of reducing Schrodinger equation by an appropriate change of the variable to form of generalized hypergeometric equation [7]. The polynomial was discovered by Sir E.J.Routh[15] and by V. I. Romanovski [16]. The notion "finite" refers to the observation that, for any given set of parameters (i.e. in any potential) only a finite of polynomials appear orthogonal.[17]

A class of hyperbolic potentials play the essential roles in interatomic and intermolecular forces ${ }^{[18]}$ and can be used to describe molecular vibrations. The Hulthen plus Eckart potential with the centrifugal term is a generalized hyperbolic form of Eckart potential, and therefore can be

Manuscript received March 9, 2011; revised April 20, 2012.

Cari is with Physics Department Sebelas Maret University (email: carinln@yahoo.com).

Suparmi is with Science Education of Post Graduate Program, Sebelas Maret University. potentially applied in describing the molecular vibration and intermolecular forces.

This paper is organized as follows. In section 1.1, we review the finite Romanovski polynomial briefly. In section 1.2, we find the bound state energy solution and wave function. A brief conclusion is presented in section 2 .

\section{A. Review of Formulas for the Polynomial Solution to the Generalized Hypergeometric Equation} [19].

The generalized hypergeometric equation is given as [18],

$$
\sigma(x) \frac{d^{2} y_{n}(x)}{d x^{2}}+\tau(x) \frac{d y_{n}(x)}{d x}+\lambda_{n} y_{n}(x)=0
$$

where $\sigma(x)=a x^{2}+b x+c ; \tau=d x+e$ and

$$
\lambda_{n}=-\{n(n-1)+2 n(1-p)\}
$$

Equation (1) is described in the textbook by NikiforovUvarov ${ }^{[9]}$ where it is cast into self adjoint form and its weight function, $w(x)$, satisfies the so called Pearson differential equation.

$$
\frac{d(\sigma(x) w(x))}{d x}=\tau(x) w(x)
$$

The weight function obtained by solving the Pearson differential equation is

$$
w(x)=\exp \left(\int \frac{(d-2 a) x+(e-b)}{a x^{2}+b x+c} d x\right.
$$

The corresponding polynomials to the weight function equation (4) are built up from the Rodrigues representation that is given as

$$
y_{n}=\frac{1}{w(x)} \frac{d^{n}}{d x^{n}}\left\{\left(a x^{2}+b x+c\right)^{n} w(x)\right\}
$$

For Romanovski polynomial, the values of parameters in equation (4) are:

$a=1, b=0, c=1, d=2(1-p)$, and $e=q$ with $p>0$

By inserting equation (6) into equation (4) we obtain the weight function as

$$
w(x)=\left(1+x^{2}\right)^{-p} e^{q \tan ^{-1}(x)}
$$

This weight function was first reported by Routh and then by Romanovski. The polynomials associated with equation (7) are Romanovski polynomials and denoted by $R_{n}^{(p, q)}(x)$. As long the weight function decreases by $x^{-2 p}$, integral of the type 


$$
\int_{-\infty}^{\infty} w^{(p, q)} R_{m}^{(p, q)}(x) R_{m^{\prime}}^{(p, q)}(x) d x
$$

will be convergent only if

$$
m^{\prime}+m<2 p-1
$$

This means that there are only a finite number of Romanovski polynomials that are orthogonal. The differential equation satisfied by Romanovski Polynomial is

$$
\begin{gathered}
\left(1+x^{2}\right) \frac{\partial^{2} R_{n}^{(p, q)}(x)}{\partial x^{2}}+\{2 x(-p+1)+q\} \frac{\partial R_{n}^{(p, q)}(x)}{\partial s}- \\
\{n(n-1)+2 n(1-p)\} R_{n}^{(p, q)}(x)=0
\end{gathered}
$$

which is hypergeometric type equation with parameters expressed in equation (6).The Romanovski polynomials obtained from equations (5) and (7) are

$$
\begin{aligned}
R_{n}^{(p, q)}(x)= & \frac{1}{\left(1+x^{2}\right)^{-p} e^{q \tan ^{-1}(x)}} \frac{d^{n}}{d x^{n}}\left\{\left(1+x^{2}\right)^{n}(1+\right. \\
& \left.\left.x^{2}\right)^{-p} e^{q \tan ^{-1}(x)}\right\}
\end{aligned}
$$

\section{B. The Eigenvalues and Eigenfuction of Hulthen Plus}

Eckart Potentials with Centrifugal Term in terms of Romanovski Polynomials

The Schrodinger equation for Hulthen [14] potential plus Eckart [13] potential with centrifugal term is

$$
\begin{gathered}
-\frac{\hbar^{2}}{2 m}\left\{\frac{d^{2} R(r)}{d r^{2}}+\frac{2}{r} \frac{d R(r)}{d r}-\frac{l(l+1) R(r)}{r^{2}}\right\}+\frac{\hbar^{2}}{2 m}\left\{V_{0} \frac{e^{\frac{-r}{a}}}{\left(1-e^{\frac{-r}{a}}\right)^{2}}-\right. \\
\left.V_{1} \frac{1+e^{\frac{-r}{a}}}{1-e^{\frac{-r}{a}}}-V_{2} \frac{e^{\frac{-r}{a}}}{1-e^{\frac{-r}{a}}}\right\} R(r)=E R(r)
\end{gathered}
$$

where $0<\frac{r}{a}<\infty, V_{0}<V_{1}, V_{0}, V_{1}$, and $V_{2}$ are positive constants.

$$
\text { If }(r)=\frac{\chi(r)}{r}, \frac{2 m}{\hbar^{2}} E=-\epsilon^{2}, \text { and } \frac{1}{r^{2}} \cong \frac{1}{4 a^{2} \sinh ^{2} \frac{r}{2 a}},
$$

then equation (12) can be rewritten as

$$
\frac{d^{2} \chi(r)}{d r^{2}}-\left\{\frac{V_{0} a^{2}+l(l+1)}{4 a^{2} \sinh ^{2} \frac{r}{2 a}}-\left(V_{1}+\frac{V_{2}}{2}\right) \operatorname{coth} \frac{r}{2 a}+\frac{V_{2}}{2}+\epsilon^{2}\right\} \chi(r)=
$$

By making an appropriate change of variable, $r=$ $f(x)=2 a \operatorname{coth}^{-1}(i x)$ in equation (14), then equation (14) becomes

$$
\begin{array}{r}
\left(1+x^{2}\right) \frac{\partial^{2} \chi}{\partial x^{2}}+2 x \frac{\partial \chi}{\partial x}- \\
\left\{V_{0} a^{2}+l(l+1)+\frac{\left(V_{1}+\frac{V_{2}}{2}\right) i x 4 a^{2}}{\left(1+x^{2}\right)}-\frac{\frac{V_{2}}{2}+\epsilon^{2}}{\left(1+x^{2}\right)} 4 a^{2}\right\} \chi=0
\end{array}
$$

To solve equation (15) in terms of Romanovski polynomial, equation (7) suggests the substitution in equation (15) as [19]

$$
\chi(f(x))=g_{n}(x)=\left(1+x^{2}\right)^{\frac{\beta}{2}} e^{\frac{-\alpha}{2} \tan ^{-1} x} D_{n}^{(\beta, \alpha)}(x)
$$

where $0<x<\infty$
By inserting equation (16) into equation (15) we obtain

$$
\begin{array}{r}
\left(1+x^{2}\right) \frac{\partial^{2} D}{\partial x^{2}}+\{2 x(\beta+1)-\alpha\} \frac{\partial D}{\partial x}- \\
\frac{\beta x \alpha-\frac{\alpha^{2}}{4}+\beta^{2}+\left(V_{1}+\frac{V_{2}}{2}\right) i x 4 a^{2}-\left(\frac{V_{2}}{2}+\epsilon^{2}\right) 4 a^{2}}{1+x^{2}}+V_{0} a^{2}+l(l+1)- \\
\left.\beta^{2}-\beta\right\} D=0
\end{array}
$$

By setting the coefficient of $\frac{1}{1+x^{2}}$ in equation (18) to be zero, that are

$$
-\frac{\alpha^{2}}{4}+\beta^{2}-\left(\frac{V_{2}}{2}+\epsilon^{2}\right) 4 a^{2}=0 \text { and } \beta \alpha+\left(V_{1}+\frac{V_{2}}{2}\right) i 4 a^{2}=
$$

then equation (17) reduces to the Romanovski equation expressed in equation (10). By comparing the parameters between equations (10) and (17) we obtain the following relation:

$$
\begin{array}{r}
V_{0} a^{2}+l(l+1)-\beta^{2}-\beta=n(n-1)+2 n(1-p) \\
2(\beta+1)=2(-p+1) \text { and } \alpha=-q
\end{array}
$$

From equation (19a) and (19b) we have

$$
\begin{gathered}
\beta=-p, \alpha=-q \\
\text { and } \beta=\beta_{n}=\sqrt{V_{0} a^{2}+\left(l+\frac{1}{2}\right)^{2}}-n-\frac{1}{2}
\end{gathered}
$$

and also from equation (18) we obtain

$$
\begin{gathered}
\alpha^{2}=-8 a^{2}\left(\frac{V_{2}}{2}+\epsilon^{2}\right) \pm 8 a^{2} \sqrt{\left(\epsilon^{2}+V_{2}+V_{1}\right)\left(\left(\epsilon^{2}-V_{1}\right)\right.} \\
\beta^{2}=\frac{-\left(V_{1}+\frac{V_{2}}{2}\right)^{2} 2 a^{2}}{-\left(\frac{V_{2}}{2}+\epsilon^{2}\right) \pm \sqrt{\left(\epsilon^{2}+V_{2}+V_{1}\right)\left(\epsilon^{2}-V_{1}\right)}}
\end{gathered}
$$

Using equation (18) and (20b) we obtain

$$
\alpha=\alpha_{n}=-\frac{\left(V_{1}+\frac{V_{2}}{2}\right) i 4 a^{2}}{\sqrt{V_{0} a^{2}+\left(l+\frac{1}{2}\right)^{2}}-n-\frac{1}{2}}
$$

Finally we obtain the energy spectra by using equations (20b) and (21b), that is

$$
\begin{array}{r}
E_{n}=-\frac{\hbar^{2}}{2 m}\left\{\frac{\left(V_{1}+\frac{V_{2}}{2}\right)^{2} a^{2}}{\left(\sqrt{V_{0} a^{2}+\left(l+\frac{1}{2}\right)^{2}}-n-\frac{1}{2}\right)^{2}}+\frac{\left(\sqrt{V_{0} a^{2}+\left(l+\frac{1}{2}\right)^{2}}-n-\frac{1}{2}\right)^{2}}{4 a^{2}}-\right. \\
\left.\frac{V_{2}}{2}\right\}
\end{array}
$$

The energy spectra in equation (23) is in agreement with the result obtained using NU method.

To determine the wave function, equations (20a), (20b) and (22) are inserted into equations (7) and (11) so that we obtain the weight function $w(x)$ and the Romanovski 
polynomials $R_{n}^{(-\beta,-\alpha)}(x)$ as

$w^{(-\beta,-\alpha)}(x)=\left(1+x^{2}\right)^{\beta_{n}} e^{-\alpha_{n} \tan ^{-1}(x)}==(1+$
$\left.x^{2}\right)^{\sqrt{V_{0} a^{2}+\left(l+\frac{1}{2}\right)^{2}}-n-\frac{1}{2}} \exp \left(\frac{4 i a^{2}\left(V_{1}+\frac{V_{2}}{2}\right)}{\sqrt{V_{0} a^{2}+\left(l+\frac{1}{2}\right)^{2}}-n-\frac{1}{2}} \tan ^{-1}(x)\right)$

And

$$
\begin{gathered}
D_{n}^{(\beta, \alpha)}(x)=R_{n}^{(-\beta,-\alpha)}(x)=\frac{1}{\left(1+x^{2}\right)^{\beta} e^{-\alpha \tan ^{-1}(x)}} \frac{d^{n}}{d x^{n}}\{(1+ \\
\left.\left.x^{2}\right)^{\beta+n} e^{-\alpha \tan ^{-1}(x)}\right\}
\end{gathered}
$$

where $\beta_{n}$ and $\alpha_{n}$ are expressed in equations (20b) and (22). As a result, the wave function of the nth level is

$$
\begin{array}{r}
\chi_{n}(f(x))=g_{n}(x)= \\
\left(1+x^{2}\right)^{\frac{\beta n}{2}} e^{\frac{-\alpha_{n}}{2} \tan ^{-1} x} R_{n}^{(-\beta,-\alpha)}(x) \frac{1}{\sqrt{\frac{d f(x)}{d x}}}
\end{array}
$$

with $\frac{d f(x)}{d x}=\frac{2 a i}{1+x^{2}}$

Since the $\beta_{n}$ and $\alpha_{n}$ parameters are n-dependence then equation (26) may not be valid ${ }^{[19]}$, and the orthogonality of the wave functions may not be the amount to the orthogonality integral of the polynomials. The orthogonality integral of the wave functions is given as

$$
\int_{0}^{\infty} \chi_{n}(r) \chi_{n^{\prime}}(r) d r=\delta_{n n^{\prime}}
$$

The implication of the orthogonality of wave functions equation (27a) is the orthogonality of the $R_{n}^{(-\beta,-\alpha)}(x)$ polynomials which is given as

$$
\int_{-\infty}^{\infty}\left(1+x^{2}\right)^{\frac{\beta n}{2}} e^{\frac{-\alpha_{n}}{2} \tan ^{-1} x}(1+
$$

22) $\beta n^{\prime} 2 e-\alpha n^{\prime} 2 \tan ^{-1} 1 x R n-\beta,-\alpha x R n^{\prime}-\beta,-\alpha x 2$ aid $x 1+x 2$

which is not finite.

By carrying out the differentiations of equation (11), we find the lowest three unnormalized polynomials to be

$$
\begin{gathered}
R_{0}^{\left(-\beta_{0},-\alpha_{0}\right)}(x)=1 \\
R_{1}^{\left(-\beta_{1},-\alpha_{1}\right)}(x)==\left(\beta_{1}+1\right) 2 x-\alpha_{1}(28 \mathrm{~b}) \\
R_{2}^{\left(-\beta_{2},-\alpha_{2}\right)}(x)=2\left(\beta_{2}+2\right)\left(2 \beta_{2}+3\right) x^{2}-2 \alpha_{2}\left(2 \beta_{2}+\right. \\
3) x+\alpha_{2}^{2}+2 \beta_{2}+4
\end{gathered}
$$

$$
\begin{array}{r}
R_{3}^{\left(-\beta_{3},-\alpha_{3}\right)}(x)=4 x^{3}\left(\beta_{3}+3\right)\left(2 \beta_{3}+5\right)\left(\beta_{3}+2\right)- \\
6 \alpha_{3} x^{2}\left(2 \beta_{3}+5\right)\left(\beta_{3}+2\right)+2 x\left(6 \beta_{3}^{2}+3 \alpha_{3}^{2} \beta_{3}+28 \beta_{3}+\right. \\
\left.6 \alpha_{3}^{2}+34\right)-2 \alpha(2 \beta+5)-\alpha\left\{\alpha^{2}+2 \beta+6\right\}
\end{array}
$$

where $\beta_{n}$ and $\alpha_{n}$ are expressed in equations (20b) and (22).

\section{CONCLUSION}

In this work we present the approximate solution of one dimensional Schrodinger equation for Hulthen potential plus Eckart potential including centrifugal term in terms of Romanovski polynomial. The energy spectrum is obtained in the closed form and the wave function obtained is expressed in terms of Romanovski polynomials. Due to ndependence of the weight function parameters, the Romanovski polynomials that are orthogonal are not finite.

\section{ACKNOWLEDGMENT}

This work is partly supported by DIPA BLU of Post Graduate grant, Sebelas Maret University contract number 2910/UN27.10/PG/2011

\section{REFERENCES}

[1] L. Infeld and T. D. Hull, Rev. Mod. Phys., no. 23, 1951.

[2] L. E. Gendenhtein, JETP Lett., no. 38,pp. 356, 1983.

[3] F. Cooper, J. N. Ginocchio, and A. Khare, Phys. Rev. D36, pp. 2458, 1987.

[4] E. Witten, Nucl. Phys. B188, pp. 513, 1981.

[5] R. Dutt, A. Khare, and U. P. Sukhatme, Am. J. Phys., vol. 56, pp. 163, 1988.

[6] H. Goudarzi and V. Vahidi, Adv. Studies Theor. Phys., vol. 5, no. 10, pp. 469,2011

[7] F. Yasuk, C. Berkdemir, and R. Berkdemir, J. Phys. A: Math. Gen, vol. 38, pp. 6579, 2005.

[8] W. Koepf and M. Masjed-Jamei, Integral Transform and Special Functions, vol. 17, pp. 559, 2006.

[9] A. F. Nikiforov and U. B. Uvarov, Special Function in Mathematical Physics, Birkhausa, Basel 1988.

[10] A. N. Ikot and L. E. Akpabio, App. Phys. Res., vol. 2,no. 2, pp. $202-$ 208, 2010.

[11] Z. Ai-Ping, Q. Wen-Chao, and L. Ya-Wen, Chin. Phys. Lett., vol. 26 no. 10, pp. 100-302, 2009.

[12] R. I. Greene and C. Aldrich, Phys. Rev., A vol. 14, pp. 23-63,1976.

[13] C. Eckart, Phys. Rev., vol. 35, pp. 1303, 1930.

[14] L. Hulthen, Ark. Mat. Astron. Fys. 28 A 1942.

[15] E. J. Routh, Proc. London Math. Soc., vol. 16,pp. 245, 1884.

[16] V. I. Romanovski, Compt. Rend. Ac. Sci., Paris, vol. 188, pp. 1023, 1929.

[17] A. Raposo, H. J. Weber, D. E. Alvarez-Castillo, and M. Kirchbach, C. Eur. Phys., J.vol. 5, pp. 253, 2007.

[18] A. N. Ikot, L. E. Akpabio, and J. A Obu, JVR, vol. 6, no. 1, pp. 1-13, 2011

[19] D. E. Alvarez- Castillo, arXiv:0808.1642v2 [ math-ph] 8 Dec. 2009. 